A Mathematical Model of the Pneumatic Force Sensor for Robot-assisted Surgery

This is the peer reviewed version of the following article:

Original:

Gaudeni, C., Prattichizzo, D. (2019). A Mathematical Model of the Pneumatic Force Sensor for Robotassisted Surgery. In Proc. 2019 IEEE World Haptics Conference (WHC) (pp.598-603). IEEE [10.1109/WHC.2019.8816087].

Availability:

This version is availablehttp://hdl.handle.net/11365/1082331

since 2020-07-01T12:18:41Z

Publisher:

IEEE

Published:

DOI:10.1109/WHC.2019.8816087

Terms of use:

Open Access

The terms and conditions for the reuse of this version of the manuscript are specified in the publishing policy. Works made available under a Creative Commons license can be used according to the terms and conditions of said license.

For all terms of use and more information see the publisher's website.

(Article begins on next page) 


\title{
A Mathematical Model of the Pneumatic Force Sensor for Robot-assisted Surgery
}

\author{
Chiara Gaudeni ${ }^{1}$ and Domenico Prattichizzo ${ }^{1,2}$
}

\begin{abstract}
Restoring the sense of touch in robotic surgery is an emerging need several researchers tried to address. In this paper, we focused on the slave side proposing a pneumatic sensor to estimate contact forces occurring during the interaction between surgical instruments and anatomical areas. It consists of a tiny pneumatic balloon, which, after being inflated, appears near the tip of the instrument during the measurement phase only. This paper presents a mathematical method relating the intensity of the contact force to the variation of pressure inside the balloon. The latter was modeled as a spherical elastic membrane, whose behavior during contact was characterized taking into account both the deformation of the membrane and the compression of the contained gas. Geometrical considerations combined with an energetic approach allowed us to compute the force of interest. The effectiveness of our sensing device has been confirmed by experimental results, based on comparison with a high-performance commercial force sensor.
\end{abstract}

\section{INTRODUCTION}

Over the past 20 years, robot-assisted minimally invasive surgery (RMIS) has been increasingly developed with the aim of improving precision, reducing errors and incision size, and facilitating recovery after surgery [1]. However, the absence of haptic feedback is still a limitation. During open procedures, clinicians have direct access to anatomical surfaces and can investigate manually their consistency to detect abnormal tissues [2]. RMIS systems have hampered those fundamental palpation procedures because of the physical separation between master and slave side. Interaction forces during robotic surgery can only be estimated by observing the deformation of the tissue on the images of the endoscopic cameras [3], but compensation by visual feedback does not prevent from inaccurate discrimination. Many studies pointed out the clear benefits of haptic feedback restoration in several clinical applications, e.g. localization of hard inclusions or vessels, soft tissue grasping, manipulation and incision, needle driving [4], and also the training phase of robotic surgeons [5].

It has been demonstrated that restoring the haptic capability in RMIS contributes to improving accuracy and safety [6]. For this reason, a great effort has been made to develop novel interfaces able to provide haptic feedback to the surgeon, but far fewer works exist regarding the design of sensing systems. Indeed, any attempts to measure or estimate forces applied during robotic surgery had to face technical challenges. Many researchers used commercially available force sensors, which are very effective to measure force accurately, but not always suitable for RMIS due to constraints in terms of

\footnotetext{
${ }^{1}$ Department of Information Engineering and Mathematics, University of Siena, Via Roma 56, 53100 Siena, Italy (gaudeni,prattichizzo) @diism.unisi.it

${ }^{2}$ Department of Advanced Robotics, Istituto Italiano di Tecnologia, Via Morego 30, 16163 Genova, Italy
}

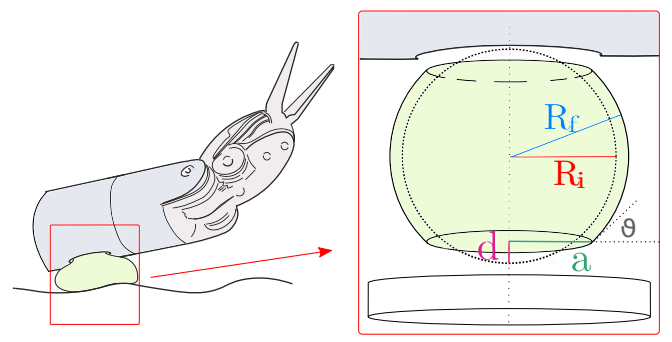

Fig. 1. Working principle and sketch of the pneumatic sensor: undeformed (dashed) and deformed (green) states of the membrane.

size, cost, biocompatibility and sterilizability [7]. Another research direction is to estimate mechanical properties and interaction forces by tracking tissue deformation, for example using deformable active contours to observe changes over time [8]. This method, however, requires the knowledge of physical parameters of human organs that are not always available. Other approaches of force measurements used light modulation techniques [9], elastomer elements [10], or combination of vision and mechanics of the spring [11].

In this paper, we present a new pneumatic-based method to estimate contact forces between instruments and tissues during robotic surgery. We pursued the idea of using a tiny pneumatic balloon developed in an earlier paper [12], by proposing a more general mathematical model.

\section{THE PNEUMATIC SENSOR}

This work extends the proof of concept presented in [12], in which we developed a pneumatic force sensor taking advantage of an air-filled balloon. The main idea is presented in Fig. 1. An elastic and sphere-shaped membrane is placed in a tiny hollow inside the surgical instrument, very close to the tip. By inflating this pneumatic balloon, it goes out from its cavity only when force measurement is required. Once it comes into contact with the human tissue, a change in the air pressure inside the balloon is registered by a pressure sensor. This value is proportional to the norm of the contact force.

We consider this approach beneficial in several ways. Primarily, the use of disposable materials already widespread in surgery, such as latex, polyurethane, or silicone, avoids sterilization and biocompatibility issues [13]. Another fundamental aspect is cost reduction. Since most of the surgical tools in RMIS are disposable, the sensing system must not represent a significant cost. Our device complies with this requirement because the pressure sensor and the electronics can be located out of the operational workspace and do not have to be replaced after every operation. Indeed, the only part that enters the human body is the membrane and 
measurement information is transferred by means of a gas to the sensitive components. Another advantage is the possibility to set the inflating pressure so as to have a sensor with different stiffnesses depending on the anatomical area. It is important to have the balloon always more compliant than the tissue so that the deformation caused by the contact occurs in the sensing system only. The location of the pneumatic sensor represents an additional benefit: it is hidden inside the body of the instrument when force measurement is not needed, so it does not limit the surgical workspace. Furthermore, the elastic membrane adapts to different shapes of tissues and can be easily miniaturized.

In this paper, we propose a novel model characterizing the sensor's behavior. The main outcome of our first work [12] was that the norm of the contact force and the resultant increase of the pressure inside the balloon were related through a quadratic function experimentally detected. The coefficients within this relation depended on the value of the pressure the balloon had been inflated to. Even though the experimental relation identified in this earlier paper was suitable to estimate the contact force, we believe that a mathematical model is necessary for further development of our sensor. Thanks to a model, calibration is not needed anymore each time size or material of any components of the pneumatic circuit change.

\section{A MATHEMATICAL MODEL}

To derive the mathematical model we assumed that:

- the considered pressure range allows us to treat the behavior of the membrane using linear elasticity theory, since deformations are reversible and relatively small;

- the shape of the inflated balloon is a sphere, whose radius is known for each value of inflating pressure;

- the tissue of interest is always stiffer than the sensor and is considered to be flat (with a radius of curvature much bigger than the radius of the balloon).

The contact between the pneumatic balloon and the human tissue was modeled as the one between a deformable, homogeneous, elastomeric, spherical membrane and a solid. Fig. 1 shows the membrane before and after the contact.

The balloon is initially inflated with a certain overpressure of gas $\Delta P_{i}$, which is the difference between the internal pressure $P_{i}$ and the atmospheric pressure $P_{0}$. Therefore, it is characterized by a radius $R_{i}$ and a membrane thickness $h$, both proportional to $\Delta P_{i}$. After the contact, due to the compression of the internal gas and the elasticity of the membrane, the pneumatic balloon adopts the shape of a sphere characterized by a wider radius $R_{f}>R_{i}$, with two flattened areas. We supposed these two contact surfaces are equivalent. The balloon balances the increased pressure $P_{f}$ by stretching, increasing surface area and diminishing $h$. Since internal pressure is equally distributed in the whole circuit, the contact force we aim to compute is

$$
F=\Delta P_{f} \pi a^{2}
$$

with $a$ the radius of the circular contact surface and $\Delta P_{f}$ the difference between $P_{f}$ and $P_{0}$. Defining as $d$ the deformation as depicted in Fig. 1, the contact radius $a$ is $\sqrt{R_{f}^{2}-\left(R_{i}-d\right)^{2}}$. Since $\Delta P_{f}$ is measured, the only unknowns are $d$ and $R_{f}$.

\section{A. State of the art in modeling deformation of spheres}

Lulevitch et al. evaluated the contact force leading to deformation of microcapsules using an energetic method and the assumption of constant volume before and after the contact, reasonable only for small deformations. The total reaction force proposed in their paper has two components (stretching and bending) and reads [14]:

$$
F=F_{\text {str }}+F_{\text {bend }}=\frac{2 \pi E h d^{3}}{(1-v) R_{i}^{2}}+\frac{\pi}{\sqrt{2}} E h^{2} \sqrt{\frac{d}{R_{i}}}
$$

where $E$ is Young's modulus and $v$ is Poisson's coefficient of the membrane. In our application, for deformations leading to a small reduction of the volume, we might neglect the compression of the balloon and assume to be in the same conditions described in [14]. An approach to compute the force of interest consists in equalizing (2) to (1). The two unknowns in this equation are $d$ and $R_{f}$, but, from volume conservation, $R_{f}$ could be approximated to $R_{i}+d^{2} /\left(2 R_{i}\right)$ [14].

Shanahan [15] proposed another formula to compute the difference between $R_{f}$ and $R_{i}$, knowing $P_{f}$ and $P_{i}$ :

$$
\Delta R=\frac{R_{i}^{2}(1-v)\left(P_{f}-P_{i}\right)}{2 E h-R_{i}(1-v)\left(P_{f}-P_{i}\right)}
$$

Also his work is limited to small deformations, but it is not based on volume conservation. Thus, another option is to use (3) in the computation of the contact radius $a$ and equalize the contact force in (1) and (2). Since (2) has not been obtained from simplifications based on volume conservation, the computation takes into account the variation of the volume of the balloon. Both these reasonings are effective, but for larger deformations the assumptions at their roots are no longer valid. For this reason, we studied a new mathematical method taking into account also the variation of volume due to the contact. The approach proposed in this paper is based on the elasticity theory combined with the evaluation of compression of the gas.

\section{B. Energetic approach}

The contact force we aim to estimate both deforms the membrane and compresses the gas contained within it. Thus, the contributions influencing the force are the stretching and the bending of the membrane, together with the compression force. The stretching energy is

$$
E_{s t r}=\frac{h}{2} \int \sigma \varepsilon d S
$$

where $\varepsilon$ is the two-dimensional strain tensor, $\sigma$ is the twodimensional stress tensor, and the integration is over the balloon's surface. The thickness of an inflated balloon depends on its radius. Since the volume of the membrane itself $\left(4 \pi R^{2} h\right)$ remains essentially constant [15], the thickness is $\left(h_{0} R_{0}^{2}\right) / R_{i}^{2}$, where $R_{0}$ and $h_{0}$ are the radius and the thickness of the deflated membrane. We supposed that $h$ remains constant during the compression. Being an isotropic spherical membrane, the relation between stress and strain tensors 


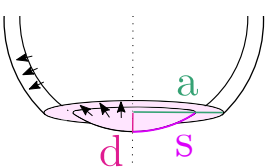

Fig. 2. Sketch of membrane while stretching. The pink area indicates the contribution of the spherical cap in low pressure cases.

is $\sigma=(E \varepsilon) /(1-v)$. To estimate the stretching energy, we made two different evaluations.

For small deformations, from the assumption that the deformed balloon has a spherical shape too, the strain is $\varepsilon=\left(R_{f}-R_{i}\right) / R_{i}$ and the stretching energy corresponds to

$$
E_{s t r} \approx \frac{4 E h \pi}{(1-v)}\left(R_{f}-R_{i}\right)^{2}
$$

Instead, to compute the stretching energy for larger deformations, we assumed that it was reasonable to split the two contributions of $i$ ) the spherical caps and $i i)$ the remaining surface of the sphere, as depicted in Fig. 2. We supposed that the two spherical caps stretch to become the two flat contact areas (pink). Defining as $s$ half the length of the cap's arc, the strain is $\varepsilon=(a-s) / s$. To simplify the computation, $s$ can be approximated to the chord $\sqrt{2 R_{i} d}$. Apart from the spherical caps, the balloon's surface stretches increasing the radius of the sphere. The resulting stretching energy is

$$
E_{s t r} \approx \frac{4 E h \pi}{(1-v)}\left[\left(\frac{R_{f}-R_{i}}{R_{i}}\right)^{2}\left(R_{i}^{2}-R_{i} d\right)+\left(\frac{a-s}{s}\right)^{2}\left(R_{i} d\right)\right]
$$

To distinguish between small and large deformations, we analyzed the initial inflating pressure value. At the same exerted force, higher pressure balloons are subject to a smaller indentation, and thereby a smaller contact area. Thus, if the inflating pressure value is higher than a certain threshold, we can assume that the balloon maintains a spherical shape. If it is smaller than the threshold, the contribution of the final flat surfaces to the stretching energy is not negligible anymore. In this case, the large contact areas are supposed to be given from the stretching of the spherical caps.

The contribution of the bending to the whole elastic energy is negligible for a thin membrane, except near the edge of the contact area. An estimation of the bending energy can be made treating the membrane locally using beam theory [16]. Then, the elastic energy of bending is

$$
E_{\text {bend }}=\frac{E I}{2} \frac{h L}{\rho^{2}}
$$

where $I=h^{3} / 12$ is the second moment of area of 'beam' cross-section, $L=2 \pi a$ is the length of the contact circle, and $\rho$ is the local radius of curvature, of order $h / \theta$, with $\theta$ the contact angle. Manipulating (6) and approximating $\left(\theta \approx \sin \theta=a / R_{f}\right)$, the bending energy is

$$
E_{\text {bend }}=\frac{E h^{2} \pi}{12} \frac{a^{3}}{R_{f}^{2}}
$$

\section{Compression of the gas}

Together with the elastic energy of the membrane, we considered also the work related to the compression of the internal gas. Since it is assumed to be ideal, the increase in free energy due to compression is

$$
\begin{aligned}
E_{\text {compr }} & =-\int_{V_{i}}^{V_{f}} \Delta P d V=-\int_{V_{i}}^{V_{f}}\left(\frac{P_{i} V_{i}}{V}-P_{0}\right) d V \\
& =-P_{i} V_{i} \ln \frac{V_{f}}{V_{i}}+P_{0}\left(V_{f}-V_{i}\right)
\end{aligned}
$$

with $V_{i}$ and $V_{f}$ the volume of the whole circuit before and after contact, respectively. $V_{i}$ is given by the sum of the volume of all the pipes, defined as $V_{c}$, and the volume of the sphere of radius $R_{i}$, defined as $V_{s i}$. The balloon after contact is modeled as a sphere of radius $R_{f}\left(V_{s f}\right)$, without two symmetrical spherical caps. Thus, $V_{\mathrm{f}}=V_{c}+V_{s f}-2 \pi H^{2}\left(R_{f}-H / 3\right)$, where $H=d+\Delta R$ is the height of the spherical cap. Pressure variation involves the whole pneumatic circuit. Hence, the contact geometry can not be described by looking only at a close neighborhood of the contact surface. However, $V_{c}$ is a constant value and it should be computed once for every identical systems.

\section{Contact force}

The total reaction force, applying Castigliano's theorem, is

$$
F=\frac{\partial}{\partial(2 d)}\left(E_{s t r}+E_{\text {bend }}+E_{\text {compr }}\right)
$$

where the unknowns are the deformation $d$ and the new incremented radius $R_{f}$. To find a relation between them, the ideal gas law can be used

$$
V_{c}+V_{s i}=\frac{P_{f}}{P_{i}}\left[V_{c}+V_{s f}-2 \pi H^{2}\left(R_{f}-\frac{H}{3}\right)\right]
$$

obtaining $R_{f}$ as a function of $d$. Then, equalizing (8) to (1) we obtain an equation where $d$ is the only unknown. Solving this equation, we can compute the contact radius $a$ and finally the reaction force from (1).

\section{Model VAlidation}

\section{A. System description}

The balloon we chose was a latex membrane $0.2 \mathrm{~mm}$ thick, embedded in a 3D-printed housing made of ABSPlus (Stratasys Inc., USA), including an opening to let the sensor come out. Natural rubber latex typically has a Poisson's coefficients of 0.5 meaning the membrane keeps a constant volume while being deformed, and a Young's modulus of $2 \mathrm{MPa}$ [17]. The pneumatic circuit was composed of an air compressor Ciao 25/185 (FNA, IT), two solenoid valves L172 2/2 G1/8 (Asco Numatics Sirai, IT), a differential pressure sensor MPXV5050DP (Freescale Semiconductor, USA), an Arduino UNO board combined with a 4 Relays Shield (Arduino, IT), and some pipes and airtight fittings to connect the different components. $V_{\mathrm{c}}$ was $12.68 \times 10^{3} \mathrm{~mm}^{3}$. To avoid blast air waves during the inflating phase, undesired pressure variations, and sensor noise, the tank air compressor was equipped with a pressure regulator, the couplings were leakproof, and pressure sensor readings were processed through a first order exponential filter. After giving the desired pressure value as input to the system, the air flow was controlled by two solenoid valves. The electronic board 


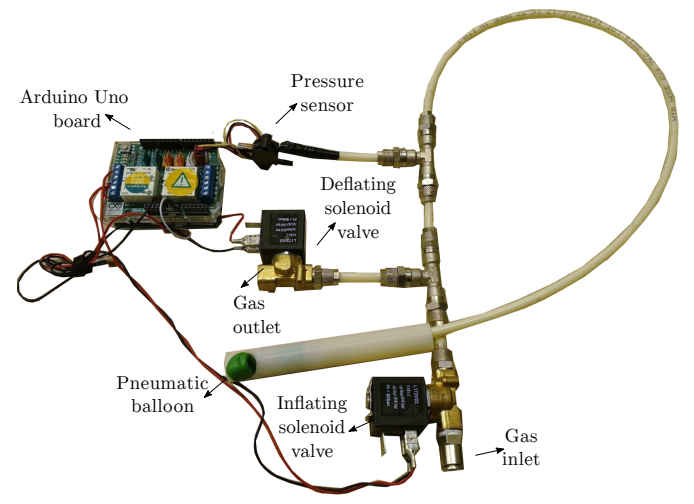

Fig. 3. Pneumatic circuit exploited in the experimental validation.

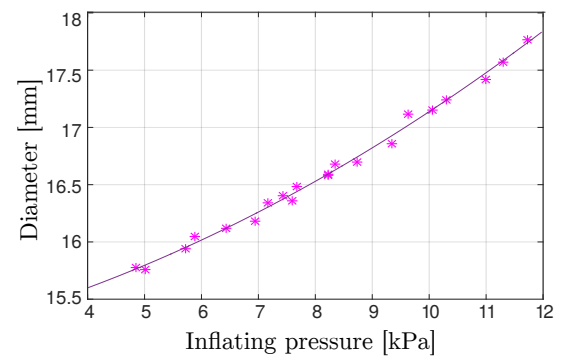

Fig. 4. Empirical relationship between diameter of the balloon and inflating pressure.

managed their opening to inflate, deflate or lock the circuit when reached one of the two thresholds, corresponding to the preset pressure value \pm the hysteresis of $0.1 \mathrm{kPa}$. The latter was introduced to prevent malfunction due to oscillations of the internal pressure when close to the chosen value. In Fig. 3 the pneumatic circuit is shown. For the scope of this paper, a simple prototype of the tool has been used.

To validate our method we conducted an experimental evaluation, comparing the estimation of the force performed by our sensor with the measures of a high resolution commercial one, the ATI Nano17 F/T sensor (ATI Industrial Automation, USA), considered as ground truth. We implemented the algorithm in MATLAB 2015b (MathWorks Inc., USA).

\section{B. Experimental evaluation}

First, the relationship between the inflating pressure $\Delta P_{i}$ and the radius of the inflated balloon $R_{i}$ had to be found. We carried out a preliminary experiment measuring the balloon diameter in 22 trials at different inflating pressures. To accurately measure its value, we used a high-precision caliber $(0.01 \mathrm{~mm}$ res.) and a magnifying glass ensuring that the contact was not affecting measurements. Then, data were interpolated to find the relation $R_{i}=\left(5.9695 \Delta P_{i}^{2}+44.652 \Delta P_{i}\right) \times 10^{-3}+7.503$. In Fig. 4 the quadratic fitting is shown. The radius of the deflated membrane $R_{0}$ was $7.503 \mathrm{~mm}$.

Then, an experiment was conducted to validate the method explored in Section III. We performed 27 trials testing different values of inflating pressure $\Delta P_{i}$, limiting the possible range to [4.95-11.45] kPa. For lower values, the membrane is so deflated that its shape under deformation can not be approximated to a sphere and its behavior is considered to

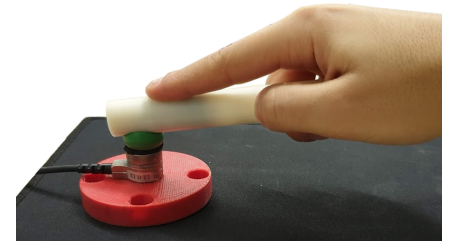

Fig. 5. Experimental procedure: the pneumatic sensor is manually pushed toward the ATI F/T sensor, considered as ground truth.

be unpredictable. On the other hand, for higher values than $12 \mathrm{kPa}$, it starts expanding more easily, increasing quickly its volume and leading to a reduction of the internal pressure.

The same procedure was repeated for each trial. At the beginning, the operator set the desired pressure value to be provided to the system. Starting from the first value of the range, the initial pressure was increased by $0.25 \mathrm{kPa}$ in each trial. For each inflating pressure value, 12 subsequent making/breaking contact actions with the ATI sensor were carried out, for a total of 324 interactions. The balloon in its housing was manually pushed toward the ATI, which in turn was rigidly attached to a flat surface (see Fig. 5). During real palpation the orientation of the sensing system will not be constrained to be always perpendicular to the anatomical surface. For this reason, we carried out manually the experimental validation, taking into account all the uncertainties due to the surgeon's behavior. For each trial we obtained a force profile as the ones depicted as examples at the bottom of Figs. 6, representing the norm of the force measured by the ATI. Although the norm corresponds approximately to the force in the user's movement direction, for the sake of accuracy we preferred not to ignore the minimum components in the other directions. The friction torque components at the contact site were negligible. As well as the pressure, the measurements collected by the ATI were processed through a first order exponential filter.

At the top of Figs. 6 the data gathered by the differential pressure sensor are depicted. For each contact action we identified $\Delta P_{i}$ as the minimum value before the pressure peak (yellow star), $\Delta P_{f}$ as the maximum (red star), and the ground truth as the ATI force peak. Because of the aim of the evaluation, i.e. validate the system in the most uncertain environment, the time elapsed between two subsequent contact actions has been chosen randomly. When faster movements were executed, after breaking contact, the pressure inside the balloon started decreasing without reaching the initial inflating value, since a new contact occurred in the meanwhile. Thus, for the sake of accuracy we did not approximate the new $\Delta P_{i}$ as the inflating value, but it was measured for each contact, and the correspondent $R_{i}$ was computed again.

In the stretching energy computation, to distinguish the two cases requiring (4) and (5) we used as threshold the pressure value of $8.85 \mathrm{kPa}$, identifying the last two fifths of the range. For higher inflating pressures, the variation of volume with respect to the initial sphere is very small. This value, computed as $\left(1-\frac{P_{i}}{P_{f}}\right) V_{i} / V_{s i}$ ranges from 0.01 to 0.18 (mean 0.095 ) for $\Delta P_{i}>8.85 \mathrm{kPa}$, while it reaches twice that amount for lower pressures. 

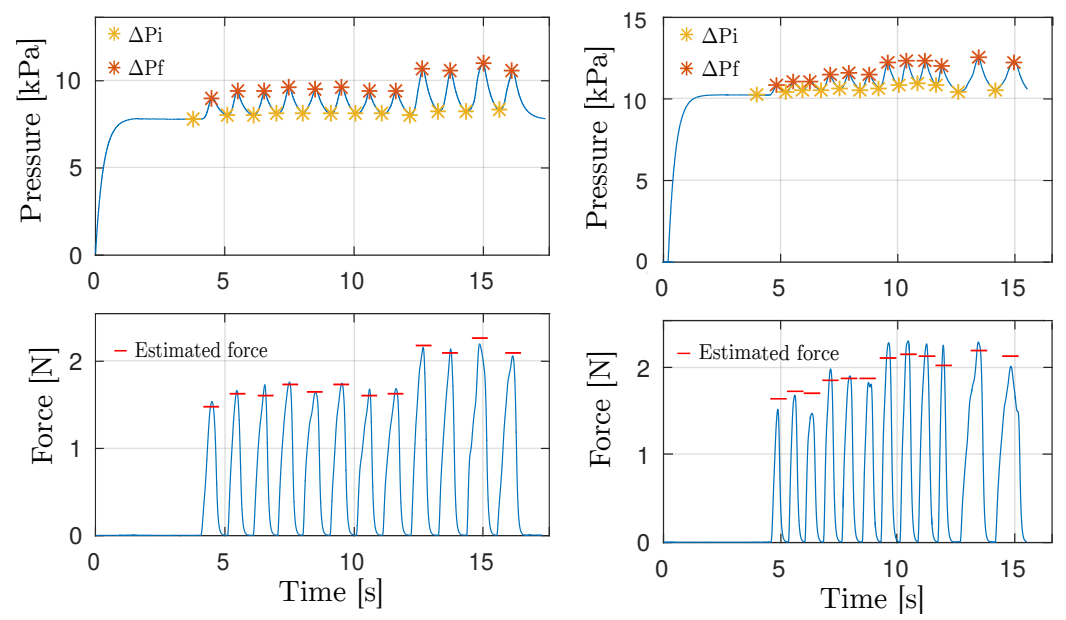

(a)

(b)
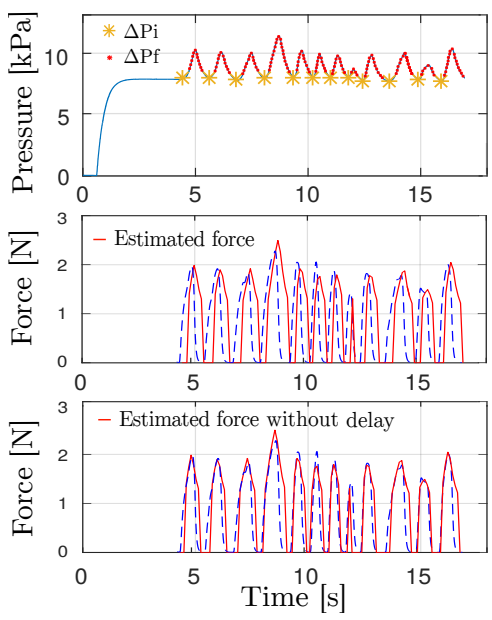

(c)

Fig. 6. On the top, the pressure measured by Freescale MPXV5050DP. The yellow stars represent the $\Delta P_{\mathrm{i}}$ values, while the red stars are the peak values reached after contact $\Delta P_{\mathrm{f}}$. On the bottom, the norm of the force measured by the ATI. The first two figures are representative trials where contact actions were executed at (a) low speed, (b) higher speed. For each contact action, the red dashes indicate the peak force values estimated by our sensor. (c) Representative trial where the contact force was estimated during the transient also. For each contact action we obtained a whole force profile.

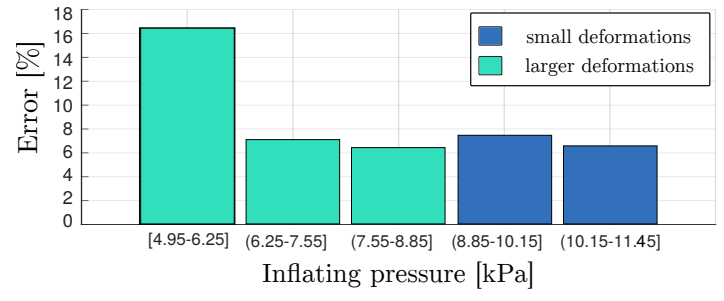

Fig. 7. Mean percentage errors in terms of pressure. Two colors have been used to indicate different procedures for the stretching energy computation.

\section{RESUlTs}

\section{A. Peak force estimation}

The red dashes in Figs. 6a and 6b represent the estimated peak force for each contact action. While the results in Fig. 6a were obtained moving slowly the sensor, in Fig. 6b a case where $R_{\mathrm{i}}$ changes time to time is shown. Also in this second condition, our system achieved promising results. The mean errors normalized over the force ground truth are (a) $2.88 \%$ and (b) $6.19 \%$. Considering the whole experiment, the mean error is $9.14 \%$. We excluded from the evaluation those trials in which the contact did not result in an appreciable variation of pressure $\left(P_{f}-P_{i}<0.5 \mathrm{kPa}\right)$. In Fig. 7 the mean percentage errors divided among five pressure subsets are depicted. The last two columns have been colored in blue to highlight they have been obtained following a different procedure for the stretching energy computation. The accuracy of our estimations clearly decreases at about $\Delta P_{i}<6 \mathrm{kPa}$.

\section{B. Comparison to other methods}

We evaluated the results obtained applying Lulevich's method (Section III-A) to those trials where the assumptions of volume's conservation and small deformations are reasonable $\left(\Delta P_{i}>8.85 \mathrm{kPa}\right)$. In this case, the mean normalized error using Lulevich's formula is $14.15 \%$. Applying Shanahan's adjustment (3), it is $14.12 \%$. Under the same conditions, our approach lead to an error of $6.77 \%$. The better performance achieved by our method is due more to the use of (9) to compute $R_{f}$ than to the small contribution of gas compression obtained from (7). In fact, stretching is the most contributing to the contact force, compression has negligible values, and the ratio between bending and stretching forces grows with the compliance of the balloon, from around $0.5 \%$ to $2 \%$.

This comparison allows us to believe our approach is meaningful. It can be used also to prove that the initial inflating pressure value is fundamental to distinguish contacts leading to small or large deformations. Using the variation of volume as the only threshold (i.e. $\Delta V / V_{i}<0.2$ ), mean errors increased to $43.88 \%$ for Lulevich's case and $52.27 \%$ for Shanahan's adjustment. It is clear that the assumption of low variation of volume is not sufficient to guarantee small deformations, while high pressure is more likely assuring a spherical shape after contact.

\section{Continuous force estimation}

To further demonstrate the usability of our sensor we tested its response in computing a continuous force profile, during the final steady-state interaction as well as the transient. The procedure followed was the same as described previously, but $\Delta P_{f}$ at each instant corresponded to the current value of internal pressure, as indicated by the red stars in Fig. 6c. As expected, the results for $\left(P_{f}-P_{i}\right)<0.5 \mathrm{kPa}$ were affected by larger errors. Excluding those values, the force profile can be reconstructed by interpolating data obtained for higher $\Delta P_{f}$ with the zero value of force before the contact. To clarify the presentation of results, the delay of the estimated force has been artificially removed introducing a time shift of $-0.12 \mathrm{~s}$, computed by aligning the peaks. As shown in Fig. 6c, the estimated force trend is similar to the ground truth, with a RMSE of $0.578 \mathrm{~N}$ most due to the low forces interpolation.

\section{DisCUSSION AND CONCLUSIONS}

This paper explores a model-based approach to estimate contact forces occurring at the slave side during robotic 
surgery. The proposed sensor consists of a pneumatic balloon placed inside the robotic instrument. In this work, we define the relationship between the contact force and the subsequent variation of pressure inside the balloon, modeled as an elastic air-filled sphere and treated using linear elasticity theory. The deformation of the membrane due to the contact with the anatomical surface has been modeled as a radius increment and the occurrence of two flattened areas. Internal pressure, displacement due to compression, and radius of the extended sphere were required to compute the reaction force. While the first value is real-time measured, to estimate indentation and radius we evaluated the forces acting on the balloon to balance the effect of the contact force, i.e. stretching, bending and compression. Moreover, the ideal gas law was used to take into account the variation of volume due to the contact. The mathematical model of the deformation was validated through experiments consisting in consecutive contact actions with a commercial force sensor, used as ground truth. The results showed agreement between model predicted and experimental data, with a mean error correspondent to the $9.14 \%$ of the real force value in the range [1-3.5] N. In addition to estimating contact force peak values, we demonstrated the feasibility of this method in continuous measurements, proving a high reliability of the system both in the transient and in the steady-state interaction.

Other approaches related the interaction force between an object and a rigid surface to the variation of some geometrical parameters, but they were limited to very small deformations and led to larger errors if compared to our method.

Compared to our previous work [12], the model-based approach here presented results to be more general, accurate and reliable. The force-pressure relationship is the result of a mathematical model and it is easy to tune against changes of components or design parameters. The only element requiring an experimental detection when size or material change is the balloon radius, but it is far simpler than a complete characterization of the system behavior empirically performed. Future development will also consider using other techniques, for example based on vision, to estimate the initial radius.

The resolution and the range of our sensor are customizable, depending on the material the membrane is made, the pressure it is inflated at, the balloon size, and the resolution of the pressure sensor. Our device has a simple mechanical miniaturizable structure. Hence, the prototype described in this paper will be able to meet the size limitations for RMIS. After miniaturization, we will properly characterize the sensor evaluating its force range and resolution at different pressure values. The use of a gas as a means to estimate interaction forces represents a major advantage in terms of delocalization of the electronics, cost, sterilizability, and biocompatibility.

However, we are aware of the limitations affecting the present study. The experimental validation we conducted relies only on tests on a rigid surface (the ATI) at the contact. For future development, experiments with softer surfaces will be considered. In addition, our pneumatic sensor is not capable of measuring very small values of force $(<1 \mathrm{~N})$ nor giving information about the direction of the applied force. Future work will involve characterizing the usability of the proposed device while displaying the estimated forces at master side. Feasibility in practical usage will be explored to investigate also if the delay due to the slightly slow dynamics might represent an issue for high quality haptic feedback. The limitations of the sensor in low pressure case will be addressed by exploring different biocompatible elastic materials. Then, one of the future objectives will be the actual integration with surgical tools. Besides our sensor was developed for robotic surgery, the sensing principle can be used to compute contact forces occurring in other teleoperation scenarios.

\section{ACKNOWLEDGMENT}

This work was supported from the European Union's Horizon 2020 Research and Innovation Programme under Grant Agreement n ${ }^{\circ} 688857$ of the project 'SoftPro'. We thank Andrea Tudico, MSc, for assistance during the development of the mathematical model.

\section{REFERENCES}

[1] A. R. Lanfranco, A. E. Castellanos, J. P. Desai, and W. C. Meyers, "Robotic surgery: a current perspective," Annals of surgery, vol. 239, no. 1 , p. $14,2004$.

[2] G. De Gersem, H. Van Brussel, and F. Tendick, "Reliable and enhanced stiffness perception in soft-tissue telemanipulation," The Int. Journal of Robotics Research, vol. 24, no. 10, pp. 805-822, 2005.

[3] P. Valdastri, M. Simi, and R. J. Webster III, "Advanced technologies for gastrointestinal endoscopy," Annual Review of Biomedical Engineering, vol. 14, pp. 397-429, 2012.

[4] L. Meli, C. Pacchierotti, and D. Prattichizzo, "Experimental evaluation of magnified haptic feedback for robot-assisted needle insertion and palpation," The Int. Journal of Medical Robotics and Computer Assisted Surgery, vol. 13, no. 4, p. e1809, 2017.

[5] J. D. Brown, J. N. Fernandez, S. P. Cohen, and K. J. Kuchenbecker, "A wrist-squeezing force-feedback system for robotic surgery training," in World Haptics Conf. (WHC), pp. 107-112, IEEE, 2017.

[6] C. R. Wagner, R. D. Howe, and N. Stylopoulos, "The role of force feedback in surgery: analysis of blunt dissection," in Proc. of Symposium on Haptic Interfaces for Virtual Environment and Teleoperator Systems (Haptics), p. 73, IEEE, 2002.

[7] A. M. Okamura, "Methods for haptic feedback in teleoperated robotassisted surgery," Industrial Robot: An International Journal, vol. 31, no. 6, pp. 499-508, 2004.

[8] Y. Luo and B. J. Nelson, "Fusing force and vision feedback for manipulating deformable objects," Journal of Field Robotics, vol. 18, no. 3, pp. 103-117, 2001.

[9] P. Polygerinos, P. Puangmali, T. Schaeffter, R. Razavi, L. D. Seneviratne, and K. Althoefer, "Novel miniature mri-compatible fiber-optic force sensor for cardiac catheterization procedures," in Int. Conf. on Robotics and Automation, pp. 2598-2603, IEEE, 2010.

[10] M. C. Yip, S. G. Yuen, and R. D. Howe, "A robust uniaxial force sensor for minimally invasive surgery," IEEE Trans. on Biomedical Engineering, vol. 57, no. 5, pp. 1008-1011, 2010.

[11] A. Faragasso, J. Bimbo, Y. Noh, A. Jiang, S. Sareh, H. Liu, T. Nanayakkara, H. A. Wurdemann, and K. Althoefer, "Novel uniaxial force sensor based on visual information for minimally invasive surgery," in Int. Conf. on Robotics and Automation, pp. 1405-1410, IEEE, 2014.

[12] C. Gaudeni, L. Meli, and D. Prattichizzo, "A novel pneumatic force sensor for robot-assisted surgery," in Int. Conf. on Human Haptic Sensing and Touch Enabled Computer Applications, pp. 587-599, 2018.

[13] J. Black, Biological performance of materials: fundamentals of biocompatibility. CRC Press, 2005.

[14] V. V. Lulevich, D. Andrienko, and O. I. Vinogradova, "Elasticity of polyelectrolyte multilayer microcapsules," The Journal of chemical physics, vol. 120, no. 8, pp. 3822-3826, 2004.

[15] M. Shanahan, "A novel test for the appraisal of solid/solid interfacial interactions," The Journal of Adhesion, vol. 63, no. 1-3, pp. 15-29, 1997.

[16] M. E. Shanahan, "Adhesion of a liquid-filled spherical membrane," The Journal of Adhesion, vol. 79, no. 10, pp. 881-891, 2003.

[17] J. Lemaitre and J.-L. Chaboche, Mechanics of solid materials. Cambridge university press, 1994. 